\title{
Contribution of Iran in COVID-19 studies: a bibliometrics analysis
}

\section{Amrollah Shamsi ${ }^{1}$ (D) Mohammad Javad Mansourzadeh $^{2}$ (D) Arash Ghazbani $^{3}$ (D) Kazem Khalagi $^{4}$ (ID)

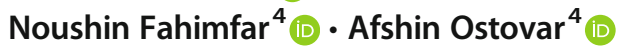

Received: 28 July 2020 / Accepted: 31 July 2020 / Published online: 14 August 2020

(C) Springer Nature Switzerland AG 2020

\begin{abstract}
Background Iran is fighting heroically against COVID-19. Due to the importance of scientific publications in better dealing with this stubborn virus, this study was conducted aiming at reviewing COVID-19 publications by Iranian scientists.

Methods We searched for COVID-19 and all its related keywords in the Web of Science (WOS), Scopus and PubMed databases to find documents published by Iranian authors until July 10, 2020. Duplicates documents were excluded, and bibliographic parameters were evaluated. Co-authorship matrix was calculated using Bibexcel, and visualizations were done using VOSviewer. Results A total of 849 documents from 3450 Iranian researchers (5.5 authors per document) were retrieved from WOS, PubMed, and Scopus and Iran ranked 12th and 13th in WOS and Scopus in terms of the number of publications. The average citation per document was 2.2 with the h-index of 18 . Original articles and letters were the most common formats for Iranian publications. The Journal of Military Medicine has published the highest number of documents. Iranian authors have mostly collaborated with researchers from the United States, Italy, the UK, and Canada, respectively. The co-occurrence network for keywords represented five publication clusters in the collection, and the largest clusters were related to epidemiological studies and public health, followed by clinical studies on COVID-19.

Conclusion Iranian researchers have had a significant scientific contribution in various areas of the disease. However, the network of studies has not been sufficiently cohesive, and more coherent collaboration between researchers at the national and international levels should be on the agenda of research policymakers in the country.
\end{abstract}

Keywords COVID-19 $\cdot$ Coronavirus $\cdot$ SARS-CoV-2 $\cdot$ Bibliometrics $\cdot$ Publications $\cdot$ Iran

\section{Introduction}

The year 2020 did not start well. With the outbreak of the new coronavirus (COVID-19) in the world, December 29, 2019 became an unforgettable day for the history of global health and the people of the world. The high prevalence of COVID19 with the possibility of its transmission from human to human [1] and its transcontinental conflict caused the World Health Organization to declare a pandemic situation in March 2020 [2]. COVID-19 causes severe respiratory and intestinal infections in humans [3] and its incubation period lasts an average of 5 days and its duration is between 4 and 7 days [4]. The main symptoms of COVID-19 include fever, cough, and shortness of breath, muscle aches, headaches, and

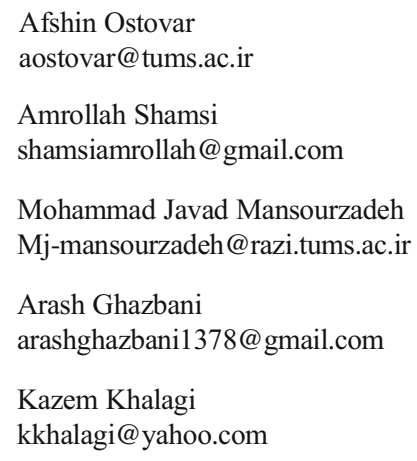

Noushin Fahimfar

nfahimfar@gmail.com

1 Bushehr University of Medical Sciences, Bushehr, Iran

2 School of Allied Medical Sciences, Tehran University of Medical Sciences, Tehran, Iran

3 Student Research Committee, Bushehr University of Medical Sciences, Bushehr, Iran

4 Osteoporosis Research Center, Endocrinology and Metabolism Clinical Sciences Institute, Tehran University of Medical Sciences, Tehran, Iran 
fatigue [5]. Having common underlying diseases such as high blood pressure, cardiovascular disease, obesity and diabetes can affect the impact of the infection and increase the mortality $[6,7]$.

According to statistics, as of July 10, 2020 brief, 12,616,578 confirmed cases, 562,039 deaths, and 7,327,360 recovered cases from 215 countries have been reported. According to the same reports, the United States, Brazil and India had the highest rates of infection, respectively, and the United States, Brazil and the United Kingdom had the highest number of deaths due to Covid-19. Iran, located in the Southwest Asian, reported its first case of Covid-19 on February 19, 2020 in Qom, and is now ranked 10th and 9th in the world with 252,720 confirmed cases and 12,447 deaths (www.worldometers.info/) due to coronavirus. The advent of COVID-19 has not only negatively affected health issues, but has also halted many economic and cultural activities around the world [8] such that the world is overshadowed by a microscopic virus.

In order to deal with COVID-19, the publication and dissemination of all scientific activities are of particular importance [9]. This led to a large number of publications on the subject of COVID-19 in a short period of time, in such a way that COVID-19 literature published since the onset of the disease has reached more than 23,000 documents, doubling every twenty days [10]. The volume of publications led to several studies to evaluate the status of scientific production on COVID- 19 and its related challenges on a global scale in bibliometric approach [11-14]. The bibliometric analysis uses literature metrology characteristics to effectively measure the contribution of an area of research, to predict detailed trends of research or hotspots in a particular field, and to make an important contribution to the prevention and treatment of diseases [13].

Each country has done its own research to answer its questions about COVID-19 in accordance with its capabilities and infrastructure. Meanwhile, Iran, as one of the countries suffering from the high incidence of COVID-19, is no exception. The scientific output of Iranian researchers is growing. According to SCImago reports, in 2019, Iran is ranked 15th in the world with the highest number of publications in the world [15]. In 2018, Iran was also ranked 31st among the top 50 countries in terms of scientific research published by top journals in Nature Index, and it has the second highest growth in this list $(26.5 \%)$ [16].

Although according to the Iran National Committee for Ethics in Biomedical Research more than 2600 research projects and 100 clinical trials are approved for COVID-19 in Iran [17], to the best of our knowledge, there have been no studies to evaluate the scientific outcomes of Iranian researchers in related with COVID-19.

Given the importance of evaluating COVID-19 research outputs in identifying the status, assisting the future decision-making, and determining the country's scientific road map in the current emergency situation, as well as the lack of studies in this area, the present study is one of the pioneering studies conducted so far with a focus on scientific productions of a particular country with a bibliometric approach. The study aimed at reviewing the general trend and scientific publications of Iran on this annoying virus and is one of the pioneers in terms of scale [18].

\section{Methods}

\section{Data source}

To identify documents related to COVID-19, a search strategy containing words related to COVID-19 and affiliation to Iran was developed on three databases: Web of Science, PubMed, and Scopus (search strategy is given in Appendix 1). Search and retrieval of data was conducted on July 10, 2020. The criteria for entering the documents into this study included all types of documents listed in the databases as mentioned above, in which at least one author with organizational affiliation to Iranian institutions was present. After extracting the documents and removing duplicate items in the Endnote software, a combined set of three databases were created. This set was used to analyze the co-authorship network and the co-occurrence network for keywords. For other scientometrics indicators that could not be reported in the form of a combined set of three databases, data obtained by the Scopus database, which is perceived as a more comprehensive and reliable bibliographic source in terms of the number of results than the other two databases, was reported.

\section{Bibliometric parameters}

In this study, the bibliometric indicators at the level of documents (number of documents, document type, number of citations, highly cited documents, average citation and hindex) and author (number of authors, average author per document, the most prolific and most highly cited author) were examined. Also, the knowledge structure of this set was analyzed at the level of social structure (co-authorship network and international cooperation network) and at the level of conceptual structure (co-occurrence network for keywords) and their network was visualized.

\section{Network visualization}

The VOSviewer v1.6.15 [19] software was used to analyze the co-occurrence network for keywords and co-authorship network. VOSviewer is a free software that can draw bibliometric 
networks at different levels of countries, organizations, journals, researchers, or individual publications using bibliographic data. Also, the text mining feature of this software has made it possible to construct and visualize the co-occurrence network for keywords.

In order to construct the co-occurrence network for keywords, the keywords of different documents were first unified in terms of various forms of writing, and then the keywords that were present in at least 5 documents were extracted, and their co-occurrence network was constructed. In order to better clarify the network, general words seen in most studies, such as demographic characteristics (male, female, child, adult, etc.) and document types (case report, systematic review, cross-sectional study, etc.) were removed from the keyword network.

For the co-authorship network, the co-authorship matrix of authors who participated in writing at least 3 documents (234 authors) was calculated by the software [10] Bibexcel V201602-20 and the VOSviewer software was used to visualize the network.

Network density was also calculated using the UCINET v6.695 software to identify the state of connectedness of the network nodes. Network density is the number of actual direct links between network nodes (authors) relative to the total number of potential connections in the network, which can be between 0 and 1 [20]. Density one means that all authors are directly related to each other, and zero density implies the isolation of authors and the absence of any connection between them.

\section{Results}

As of July 10, 2020, 382 documents have been indexed on the Web of Science, 638 on the Scopus, and 631 on the PubMed databases. Out of 1651 documents retrieved in the three databases, 802 duplicate documents were removed from the set, and finally 849 documents were identified with the participation of 3450 independent authors and were selected for the analysis of co-authorship and co-occurrence network for keywords. Of the 638 documents by Iranian authors listed on the Scopus, the original articles and letters each account for $38.9 \%$ and $33.2 \%$ of the publications, respectively, and the rest of the documents were published in the form of editorials, research notes, and short reviews. Table 1 shows the characteristics of documents by Iranian authors published on COVID-19.

In total, Iranian studies in the field of COVID-19 received 1427 citations in the Scopus database. The average citation per document in this set was 2.2 and the h-index was 18 . Table 2 shows a list of ten most frequently cited documents, along with the number of citations and their document type.
Table 1 Iranian COVID-19 documents characteristics

\begin{tabular}{ll}
\hline Description & Finding \\
\hline No. of documents & \\
$\quad$ Documents in WOS & 382 \\
Documents in PubMed & 631 \\
Documents in Scopus & 638 \\
Total Documents (After duplicates removed) & 849 \\
Authors & \\
Total Authors & 4706 \\
Avg. Authors per Document & 5.5 \\
Document Type (Scopus) & \\
Article & $248(38.8 \%)$ \\
Letter & $212(33.2 \%)$ \\
Review & $104(16.3 \%)$ \\
Editorial & $38(5.9 \%)$ \\
Note & $34(5.3 \%)$ \\
Short Communication & $2(0.3 \%)$ \\
Citation (Scopus) & \\
Total Citations & 1427 \\
Avg. Citations per Documents & 2.2 \\
H-index & 18 \\
\hline
\end{tabular}

We found that Rezaei N with 24, Haseli S with 15 and Tabarsi $P$ with 10 documents were the most active Iranian authors in the field of COVID-19 studies. The list of 10 most prolific COVID-19 research authors in terms of the number of documents and citations is presented in Table 3.

Among the medical journals hosting research related to COVID 19, the Journal of Military Medicine published the most significant number of documents. Table 4 shows a list of ten journals with the highest number of documents related to Covid-19 along with the subject area, their quartile, and the number of citations received from COVID-19 related documents.

In terms of the number of publications, Iran ranked 12th in the Web of Science database. The largest share in the publication of documents was by researchers from the United States and China. According to the Scopus, Iran ranked 13th and US researchers with 100, Italy with 42, the UK with 33 and Canada with 32 documents had the most collaboration with Iranian researchers (see Fig. 1).

The authorship distribution pattern indicated that $68.6 \%$ (582) of documents were written in small groups of 1 to 5 authors, $23.3 \%$ (198) in groups with 6 to 10 authors and $8.1 \%$ (69) in groups with more than 10 authors. The average number of authors per document was calculated 5.5. The co-authorship network of published documents is shown in Fig. 2. In this network, 234 


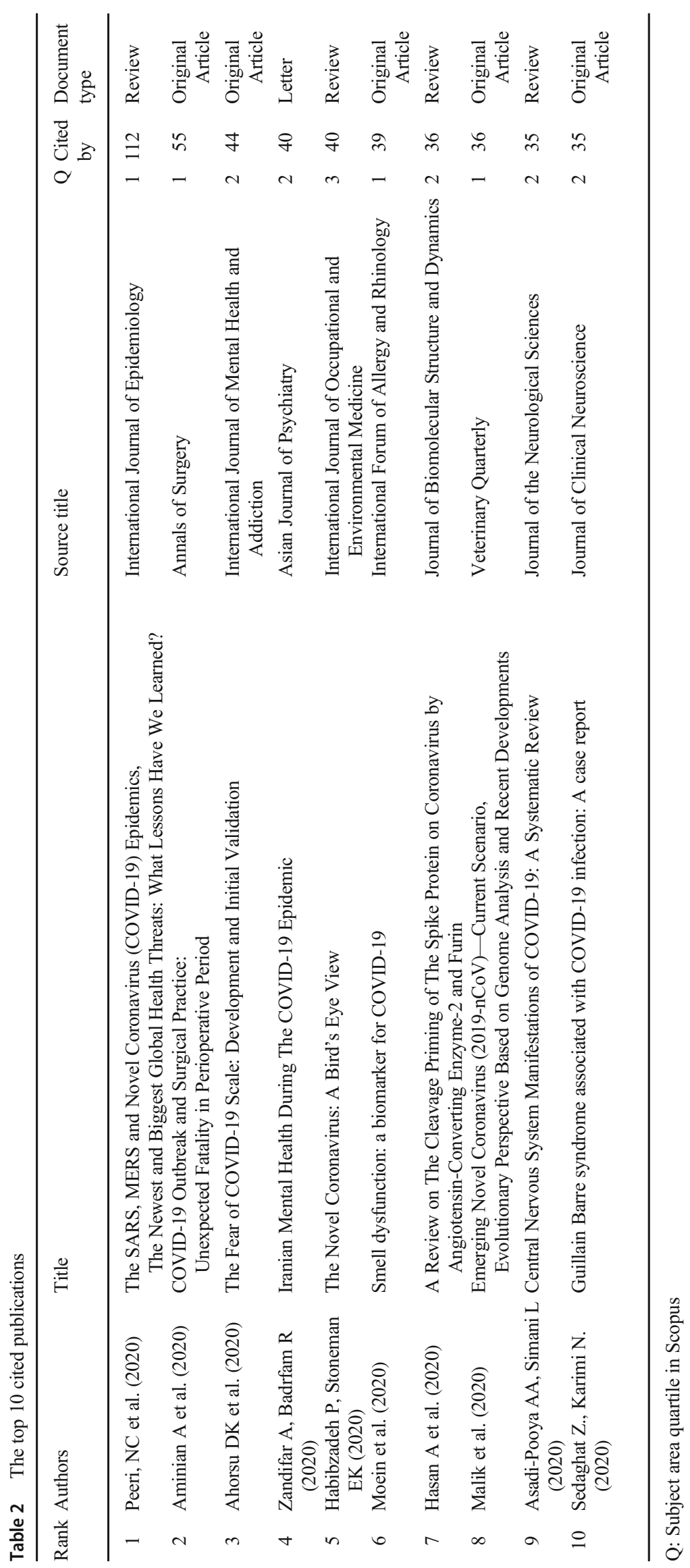


Table 3 Top 10 Prolific authors in Iranian COVID-19 studies in terms of number of records and total citation (Scopus)

\begin{tabular}{llllll}
\hline Rank & Authors & No. of records & \% of 849 & Authors & T. Citation \\
\hline 1 & Rezaei N & 24 & 2.8 & Aghamohammadi N \\
2 & Haseli S & 15 & 1.8 & Baghbanzadeh M & 112 \\
3 & Tabarsi P & 10 & 1.2 & Rezaei N & 69 \\
4 & Dadkhahfar S & 9 & 1.1 & Dadar M & 69 \\
5 & Taheri MS & 9 & 1.1 & Safari S & 60 \\
6 & Goldust M & 9 & 1.1 & Aminian A & 58 \\
7 & Lotti T & 9 & 1.1 & Karimi N & 57 \\
8 & Saffaei A & 9 & 1.1 & Ghorbani M & 55 \\
9 & Sadoughifar R & 9 & 1.1 & Falahati M & 47 \\
10 & Badrfam R & 8 & 0.9 & Sharifi M & 47 \\
\hline
\end{tabular}

authors were connected to each other by 1208 links and form a network with a density of 0.022 . The network consisted of a more extensive network with 156 authors $(66.66 \%)$ and 23 small components (sub-network).

The co-occurrence network for keywords, shown in Fig. 3, consisted of five intertwined clusters of keywords, the topics of which were:

Cluster 1 (Red): With 21 keywords such as "pandemic", "epidemiology", "disease outbreak", and "public health", the cluster showed epidemiological and public health studies on COVID-19.

Cluster 2 (Green): With 19 keywords such as "remdesivir", "chloroquine", "immunotherapy", "disease transmission" showed clinical studies, signs and symptoms of the disease, and pharmacological and nonpharmacological interventions for COVID-19.

Cluster 3 (Blue): With 13 keywords such as "Pneumonia", "mortality", "Virus detection", "PCR", "fever", showed signs and symptoms of the disease, clinical consequences and diagnostic methods for COVID19.

Cluster 4 (Yellow): With 8 keywords such as "COVID19", "Coronavirus", "SARS-COV-2", etc., mostly represented the documents introducing the virus that caused COVID-19 disease.

Cluster 5 (Purple): The smallest network cluster, with 3 keywords, including "cancer", "hypertension" \& "diabetes mellitus", showed evidence of the role of underlying diseases associated with COVID-19.

\section{Discussion}

By the time of this study, 849 documents contributed by 4706 Iranian authors were retrieved from three databases of Scopus,

Table 4 The 10 most published journals

\begin{tabular}{|c|c|c|c|c|c|c|c|}
\hline Rank & Sources & Subject Area & Q & CiteScore & NP & Citation & $\begin{array}{l}\text { Avg. } \\
\text { CPP }\end{array}$ \\
\hline 1 & Journal of Military Medicine & $\begin{array}{l}\text { Public Health, Environmental and Occupational } \\
\text { Health }\end{array}$ & 4 & 0.5 & 22 & 26 & 1.2 \\
\hline 2 & Academic Radiology & Radiology, Nuclear Medicine and Imaging & 2 & 3.9 & 17 & 19 & 1.1 \\
\hline 3 & Infection Control and Hospital Epidemiology & Infectious Diseases & 2 & 4.5 & 17 & 21 & 1.2 \\
\hline 4 & Iranian Journal of Public Health & $\begin{array}{l}\text { Public Health, Environmental and Occupational } \\
\text { Health }\end{array}$ & 4 & 0.9 & 16 & 6 & 0.4 \\
\hline 5 & Archives of Clinical Infectious Diseases & Critical Care and Intensive Care Medicine & 3 & 1.0 & 15 & 15 & 1.0 \\
\hline 6 & Archives of Medical Research & General Medicine & 1 & 3.3 & 13 & 18 & 1.4 \\
\hline 7 & Archives of Iranian Medicine & General Medicine & 1 & 2.2 & 12 & 32 & 2.7 \\
\hline 8 & $\begin{array}{l}\text { Medical Journal of The Islamic Republic of } \\
\text { Iran }\end{array}$ & General Medicine & 2 & 0.9 & 12 & 7 & 0.6 \\
\hline 9 & Medical Hypotheses & General Medicine & 1 & 2.2 & 11 & 8 & 0.7 \\
\hline 10 & Dermatologic Therapy & Dermatology & 2 & 1.8 & 10 & 28 & 2.8 \\
\hline
\end{tabular}

Q: Subject area quartile in Scopus. NP: Number of publications. Avg. CPP: Average Citations per Publication 


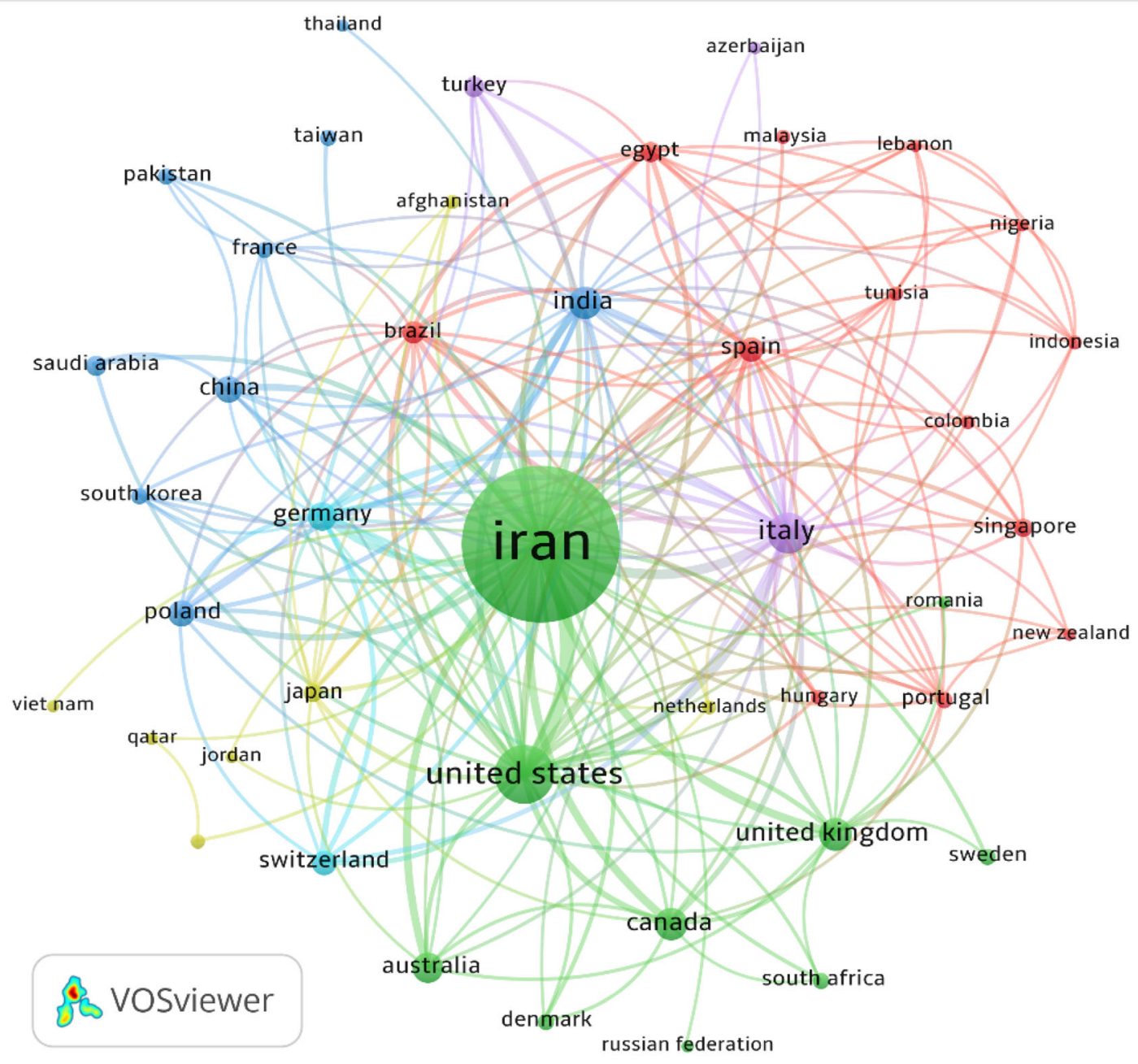

Fig. 1 Iran international collaboration map of documents indexed in Scopus

PubMed and Web of Science. Iran ranked 12th and 13th, respectively, in terms of the number of publications in the Web of Science and Scopus. The average number of authors per document was about 5.5, and original articles and letters, each having a share of about $39 \%$ and $33 \%$ respectively, was the most common format for Iranian publications. The average citation for each retrieved document was 2.2 and their hindex was 18.

The results of the study showed that after "original article", the "letter" was one of the main document types published in the field of COVID-19 researches [8, 14]. The high prevalence of COVID-19 and global struggles to detect unknowns about the virus appear to have prompted researchers to select the letter as the shortest and fastest way to disseminate scientific information. However, since more time is needed to complete studies in the field of COVID-19, original articles in the peer review process are usually published later. Reassessment after a sufficient period of time can better reflect the activities of researchers in this field.
In our study, the average citation per document on Scopus was 2.2 , and the h-index was 18 , which was slightly higher than the global average in the field of COVID-19 [21]. However, according to the published results, in the section of highly cited documents, the citation status of documents published by Iranian researchers seems to be acceptable compared to other studies $[18,22]$. It should be noted that the selection of high-quality journals can lead to high visibility and impact [23] and thus, the produced knowledge could optimally cope with the COVID-19 disease.

Due to the severe shortage of research budgets, Iranian researchers are limited in their choice of open access journals [24]. On the other hand, some medical journals, in addition to unilateral US sanctions, avoid accepting manuscripts submitted by Iranian researchers, directly or indirectly [25]. Therefore, researchers have published most of their documents in Iranian journals. Further investigation revealed that most of the citations are received by review articles. This is while the original articles of COVID-19 are more popular worldwide than other types of documents in terms of citation 


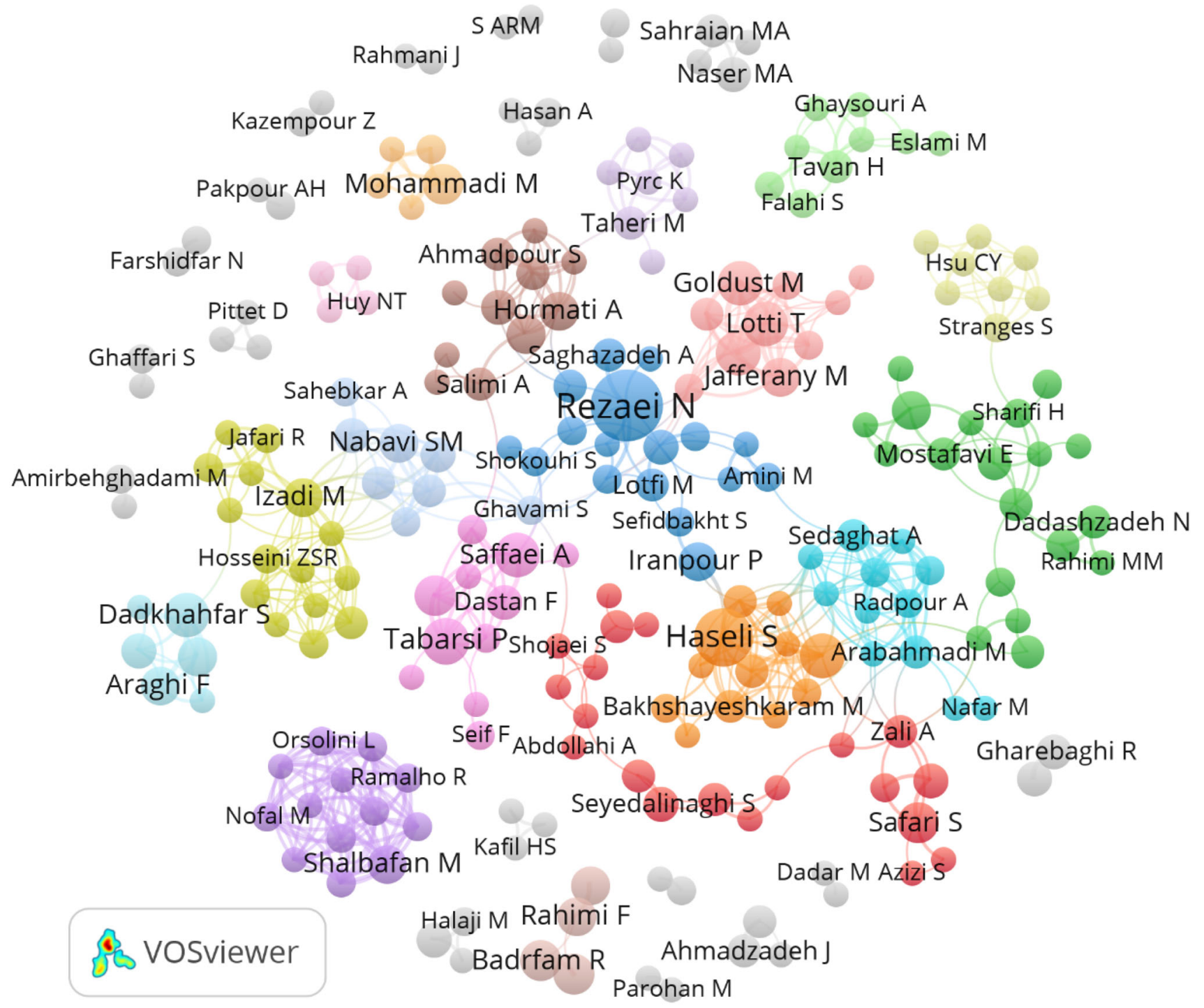

Fig. 2 Co-authorship network of Iranian researchers on COVID-19

[22]. Therefore, despite the value of other forms of document types, writing original articles can contribute more to the advancement of science.

A review of the most prolific journals showed that most of them are from domestic publications and are in the top journals in their field. Only a small number of top documents contributed by Iranian researchers were published in top journals in the field of medical sciences, and only in a few of them the corresponding author was Iranian. It is recommended that researchers identify and select journals with more visibility indicators. However, Iranian researchers have attempted to exchange and access each other's opinions in the field of COVID-19 by establishing scientific collaborations with researchers from prominent countries, such as the United States and Italy [14]. Strengthening international collaborations can help researchers achieve effective results. Therefore, identifying the factors that strengthen or weaken scientific collaboration should be on the agenda of research policymakers in the country.

Our study showed that the COVID-19 network of Iranian researchers with a density of 0.022 suffers from low coherence. This density means that the authors of COVID-19related studies used only $2.2 \%$ of the potential links between the authors, so the authors on this network have a low level of connection with each other, and their maximum capacity is not used in the network. Research teams independently conducted COVID-19 studies in Iran in 24 small sub-networks. This may be due to the heterogeneous incidence of the disease in different parts of the country. Also, most of the research was done in small scientific groups of less than 5 authors. However, compared to COVID-19 studies in the world [21], Iranian studies have larger teams. Given the current state of emergency and the impact of group collaboration on the quality of publications [26], researchers should be encouraged to 


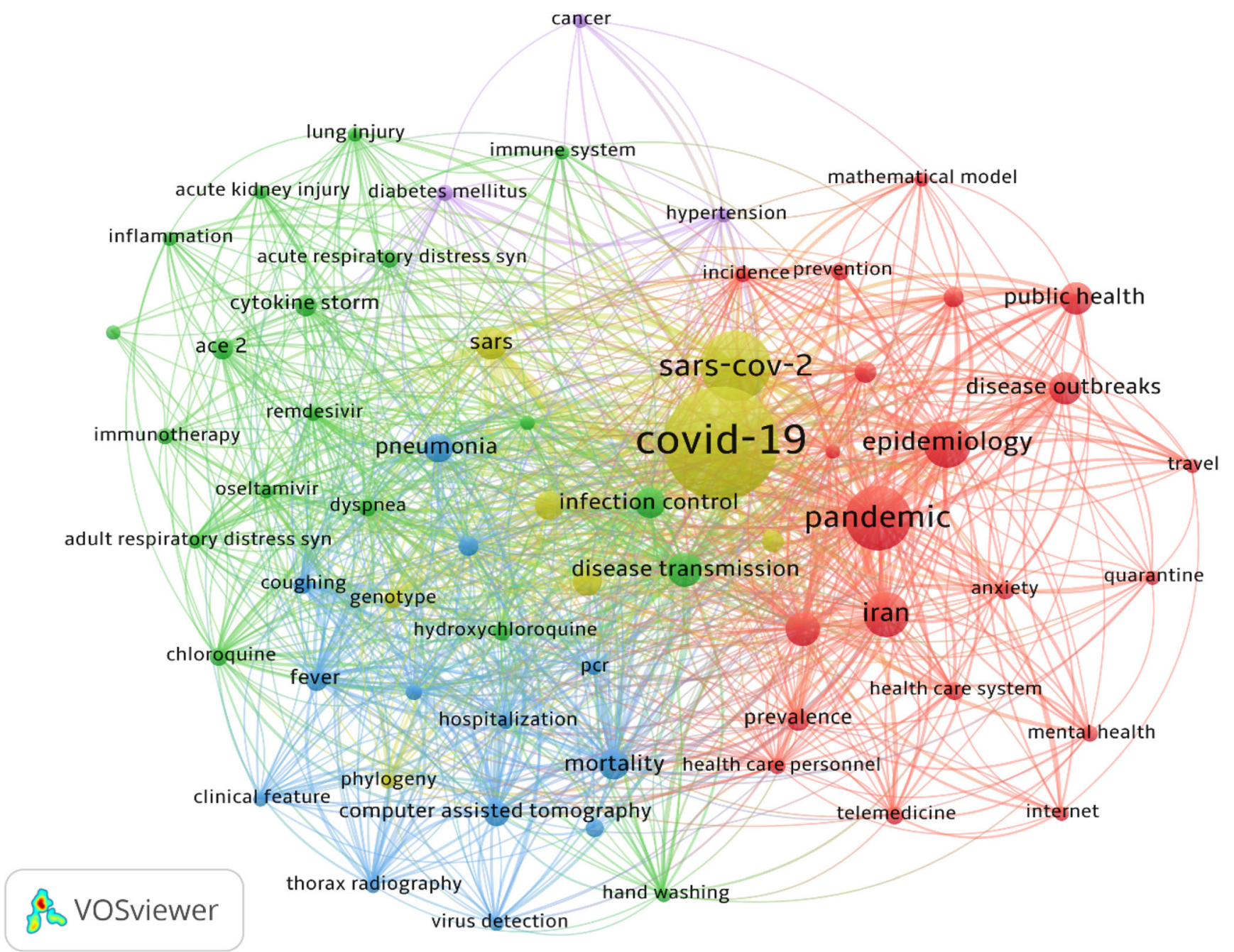

Fig. 3 Co-occurrence of keywords in documents related to COVID-19 published by Iranian researchers

increase collaboration by increasing their connection with network members. The formation of more cohesive research teams and broader research networks can lead to the promotion of publications, which is possible through research policy making by higher authorities.

Evaluation of the co-occurrence network for keyword showed that researches by Iranian authors have covered a wide range of COVID-19-related areas, including: epidemiology, pharmaceutical and non-pharmaceutical interventions, clinical aspects including disease symptoms, diagnostic methods, virology, and underlying diseases. Despite the diversity of the virus's faces, studying in other areas should not be neglected to understand it further.

\section{Strengths and limitations}

In this study, an investigation of research documents published in the subject area of COVID-19 was carried out at the national level, which has rarely been done in such a scale. In addition, we searched for data from three reliable Web of
Science, Scopus, and PubMed databases that show the scope of the work. Although this approach has covered a large portion of COVID-19 publications by Iranian researchers, but some publications by Iranian authors indexed in National (Persian) databases, Preprint databases (such as medRxiv and bioRxiv), and those articles that have yet to be published have not been retrieved in the present research, which is one of the limitations of this study.

\section{Conclusion}

Iranian researchers have made significant contributions to the publication of documents on COVID-19, and a variety of areas of the disease have been covered by them. However, the co-authorship network of studies is not sufficiently coherent. Therefore, a more consistent collaboration of research networks in the country and strengthening international collaboration should be on the agenda of research policymakers in the country. 


\section{Compliance with ethical standards}

Conflict of interest disclosures The authors declare no conflict of interest.

\section{Appendix 1: Search Strategy}

\begin{tabular}{|c|c|c|c|}
\hline Database & Search strategy & $\begin{array}{l}\text { Execution } \\
\text { date }\end{array}$ & $\begin{array}{l}\text { No. of } \\
\text { records }\end{array}$ \\
\hline PubMed & 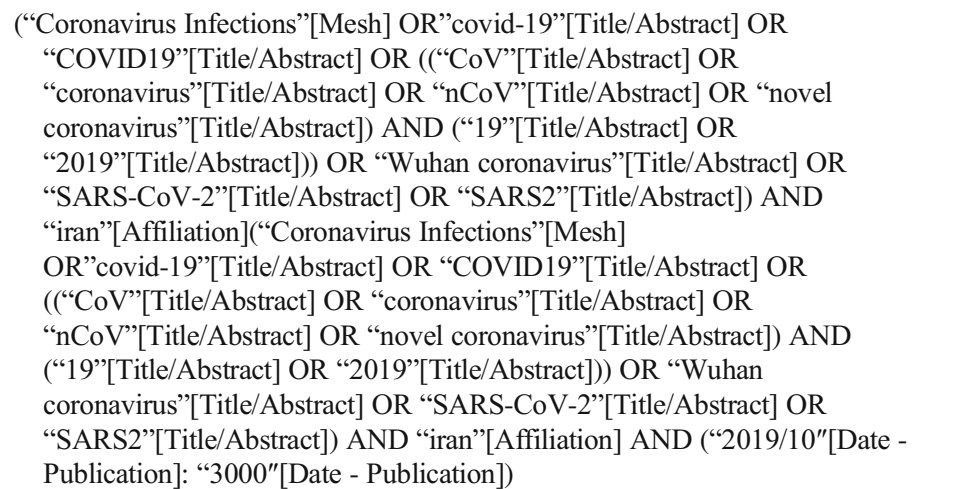 & $\begin{array}{l}10 \\
\text { July } 2020\end{array}$ & 631 \\
\hline $\begin{array}{l}\text { Web of Science } \\
\text { (Indexes: SCI-EXPANDED, SSCI, } \\
\text { A\&HCI, CPCI-S, CPCI-SSH, ESCI.) }\end{array}$ & 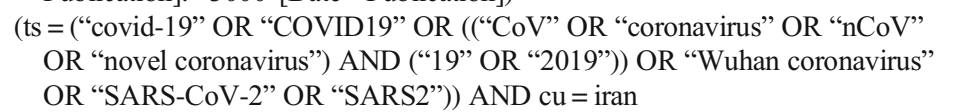 & 10 & 382 \\
\hline Scopus & $\begin{array}{l}\text { TITLE-ABS-KEY ((“covid-19” OR “COVID19” OR ((“CoV” OR “coronavirus" } \\
\text { OR "nCoV” OR “novel coronavirus") AND (“19” OR “2019”)) OR “Wuhan } \\
\text { coronavirus" OR “SARS-CoV-2” OR "SARS2”)) AND AFFILCOUNTRY } \\
\text { (iran) AND PUBYEAR > } 2018\end{array}$ & $\begin{array}{l}10 \\
\text { July } 2020\end{array}$ & 638 \\
\hline
\end{tabular}




\section{References}

1. Lancet T. Emerging understandings of 2019-nCoV. Lancet. 2020;395(10221):311.

2. Organization WH. WHO announces COVID-19 outbreak a pandemic. 2020. 2020.

3. Bonilla-Aldana DK, Quintero-Rada K, Montoya-Posada JP, Ramírez-Ocampo S, Paniz-Mondolfi A, Rabaan AA, et al. SARS-CoV, MERS-CoV and now the 2019-novel CoV: have we investigated enough about coronaviruses? - a bibliometric analysis. Travel Med Infect Dis. 2020;33:101566.

4. Li Q, Guan X, Wu P, Wang X, Zhou L, Tong Y, et al. Early transmission dynamics in Wuhan, China, of novel coronavirusinfected pneumonia. N Engl J Med. 2020;382(13):1199-207.

5. Chen N, Zhou M, Dong X, Qu J, Gong F, Han Y, et al. Epidemiological and clinical characteristics of 99 cases of 2019 novel coronavirus pneumonia in Wuhan, China: a descriptive study. Lancet. 2020;395(10223):507-13.

6. Richardson S, Hirsch JS, Narasimhan M, Crawford JM, McGinn T, Davidson KW, et al. Presenting characteristics, comorbidities, and outcomes among 5700 patients hospitalized with COVID-19 in the New York City area. JAMA. 2020;323(20):2052-9.

7. Zhou F, Yu T, Du R, Fan G, Liu Y, Liu Z, et al. Clinical course and risk factors for mortality of adult inpatients with COVID-19 in Wuhan, China: a retrospective cohort study. Lancet. 2020;395(10229):1054-62.

8. Haghani M, Bliemer MCJ, Goerlandt F, Li J. The scientific literature on coronaviruses, COVID-19 and its associated safety-related research dimensions: a scientometric analysis and scoping review. Saf Sci. 2020;129:104806.

9. Song P, Karako T. COVID-19: real-time dissemination of scientific information to fight a public health emergency of international concern. Biosci Trends. 2020;14(1):1-2.

10. Brainard J. Scientists are drowning in COVID-19 papers. Can new tools keep them afloat: Science; 2020.

11. Hamidah I, Sriyono S, Hudha MN. A Bibliometric analysis of Covid-19 research using VOSviewer. IJoST. 2020;5(2):34-41.

12. Mao X, Guo L, Fu P, Xiang C. The status and trends of coronavirus research: A global bibliometric and visualized analysis. Medicine. 2020;99(22).
13. Tao Z, Zhou S, Yao R, Wen K, Da W, Meng Y, et al. COVID-19 will stimulate a new coronavirus research breakthrough: a 20-year bibliometric analysis. Ann Transl Med. 2020;8(8):528.

14. Zhai F, Zhai Y, Cong C, Song T, Xiang R, Feng T, et al. Research Progress of coronavirus based on Bibliometric analysis. Int $\mathrm{J}$ Environ Res Public Health. 2020;17(11):3766.

15. SCImago [Portal]. SJR - SCImago Journal \& Country Rank. n.d. [cited $2020 \mathrm{Jul} 10]$. Available from: https://www.scimagojr.com

16. Crew B. The top 10 countries for scientific research in 2018. Nature Index 2019.

17. National Committee for Ethics in Biomedical Research of Iran. Available at: https://ethics.research.ac.ir. Accessed 10 July 2020.

18. Raju NV. Indian Publications on SARS-CoV-2: A Bibliometric Study of WHO COVID-19 Database. medRxiv. 2020.

19. van Eck NJ, Waltman L. Software survey: VOSviewer, a computer program for bibliometric mapping. Scientometrics. 2010;84(2):523-38.

20. Perez C, Germon R. Chapter 7 - graph creation and analysis for linking actors: application to social data. In: Layton R, Watters PA, editors. Automating open source intelligence. Boston: Syngress; 2016. p. 103-29.

21. Arias-Chávez D, Ramos-Quispe T, Villalba-Condori K, PostigoZumarán JE. World scientific production on covid-19: analysis of the period February-march, 2020. J Crit Rev. 2020;7(9):64-9.

22. Dehghanbanadaki H, Seif F, Vahidi Y, Razi F, Hashemi E, Khoshmirsafa M, et al. Bibliometric analysis of global scientific research on coronavirus (COVID-19). Med J Islam Repub Iran. 2020;34(1):354-62.

23. Lou J, Tian S, Niu S, Kang X, Lian H, Zhang L, et al. Coronavirus disease 2019: a bibliometric analysis and review. Eur Rev Med Pharmacol Sci. 2020;24(6):3411-21.

24. Butler D. How US sanctions are damaging science in Iran. Nature. 2019;574:13-4.

25. Akhondzadeh S. Possibility for science without borders in the trump era. Lancet. 2019;393(10170):405-6.

26. Geraei E, Mazaheri E, Karimi M. Intradepartment scientific collaboration in journal of research in medical sciences: a co-authorship study. J Res Med Sci. 2018;23:97.

Publisher's note Springer Nature remains neutral with regard to jurisdictional claims in published maps and institutional affiliations. 